\title{
Evaluation of phase-frequency instability when processing complex radar signals
}

\author{
Juliy Boiko ${ }^{1}$, Lesya Karpova ${ }^{2}$, Oleksander Eromenko ${ }^{3}$, Yevhen Havrylko ${ }^{4}$ \\ 1,2Department of Telecommunications and Radio Engineering, Khmelnytsky National University, Ukraine \\ ${ }^{3}$ Department of Physics and Electrical Engineering, Khmelnytsky National University, Ukraine \\ ${ }^{4}$ Department of Automation of Projection of Power Processes and Systems, National Technical University of Ukraine, \\ Igor Sikorsky Kyiv Polytechnic Institute, Ukraine
}

\begin{tabular}{|c|c|}
\hline Article Info & ABSTRACT \\
\hline Article history: & A new radar system for digital signal processing before detection is \\
\hline Received Oct 19, 2019 & proposed. These are the guidelines for selecting an intermediate frequency \\
\hline Revised Feb 5, 2020 & echo-signal selection by the features of the correlation properties of their \\
\hline Accepted Feb 25, 2020 & complex bypass are described. This paper presents the study of ambiguity \\
\hline & function $(\mathrm{AF})$ when processing complex radar signals. In this work, the AF \\
\hline Keywords: & the presence of phase-frequency instability. The received result enhances \\
\hline Ambiguity function & the potentials for distinguishing an incoherent radar signal. The numerical \\
\hline Frequency resolution & components in the complex AF, depending on the laws of the distribution of \\
\hline Radar signal & amplitude and frequency fluctuations and their parameters, allowed to get \\
\hline Signal processing & the gain in the width of the main lobe from the units to tens of times. Paper \\
\hline Tracking capability & $\begin{array}{l}\text { represents original analytical expressions for } \mathrm{AF} \text { of radio-signals modulated } \\
\text { by narrow band random processes with various distribution laws. }\end{array}$ \\
\hline
\end{tabular}

Copyright @ 2020 Institute of Advanced Engineering and Science. All rights reserved.

\section{Corresponding Author:}

Juliy Boiko,

Department of Telecommunications and Radio Engineering,

Khmelnytsky National University,

11, Instytuts'ka str., Khmelnitsky, 29016, Ukraine

Email: boiko_julius@ukr.net

\section{INTRODUCTION}

There are methods for the efficiency assessing of radar signals due to energy parameters - energy, power, spectral distribution of energy and power, amplitude, etc. [1, 2] as well as methods of efficiency estimating due to differentiating properties - the signal basis, the correlation function [3-5]. Such methods give an opportunity to evaluate the efficiency effect of just one or more indicators. This approach is low-efficient when solving the synthesis problem of optimal radar signals. In the process of development of the radio systems and signals theory a comprehensive assessment of the radar signals efficiency in the AF form was substantiated. Such a function is a two-dimensional correlation function of a complex envelope of a radar signal [4]. In the case of set initial conditions, this function defines a complete characteristic of the efficiency of radar signals.

The problem of AF radar signals synthesizing by their non-deterministic models isn't practically solved $[2,6]$. This task becomes urgent as it is possible to implement the signal processing devices with dynamic characteristics that are capable of adaptation as needed [1,7]. The non-deterministic signals can be considered for their potential capability to achieve the maximum gain under these conditions. The paper proposes analytical expressions and AF signals took account of non-deterministic components. The results of the AF radar signals research for the determination of the separation efficiency of Gaussian signals and 
linear-frequency modulated (LFM) are presented. Pointed signals represent the most characteristic class of AF signals complex, which allows to obtain uncorrelated measurements of range and speed.

\section{RESEARCH METHOD}

\subsection{The transformation of ambiguity function with regard to phase frequency instability}

The synthesis of AF radar-probing signals by non-determined models will be carried out taking into account signal parameters in the form of their density functions. AF in the form of a module of a two-dimensional correlation function of a signal complex envelope is:

$$
\Psi(\tau, F)=\frac{\left|\int_{-\infty}^{\infty} U(t) U \cdot(t-\tau) e^{j 2 \pi F t} d t\right|}{\int_{-\infty}^{\infty}|U(t)|^{2} d t}
$$

For a signal with envelope:

$$
U(t)= \begin{cases}1, & |t| \leq \frac{\tau_{i}}{2} \\ 0, & |t|>\frac{\tau_{i}}{2}\end{cases}
$$

where $\tau_{i}$ is the duration of the pulse. It is occurred:

$$
\Psi(\tau, F)= \begin{cases}\left|\sin \left[\pi F\left(\tau_{i}-|\tau|\right)\right] / \pi F \tau_{i}\right|, & |t| \leq \frac{\tau_{i}}{2} \\ 0, & |t|>\frac{\tau_{i}}{2}\end{cases}
$$

Figure 1 shows a horizontal slice of the AF surface $\Psi(\tau, F)$ at 0.5 level (darkened) and at a level which is closed to zero (shaded). Figure 2(a,b) shows vertical profiles of the surface $\Psi(\tau, F)$ by $F=0$ and $\tau=0$ planes. Apparently, they are symmetric in respect of the slice surfaces. The vertical profile $\tau=0$ as shown in Figure 2(b), is a Fourier transform of the envelope square of the rectangular pulse.

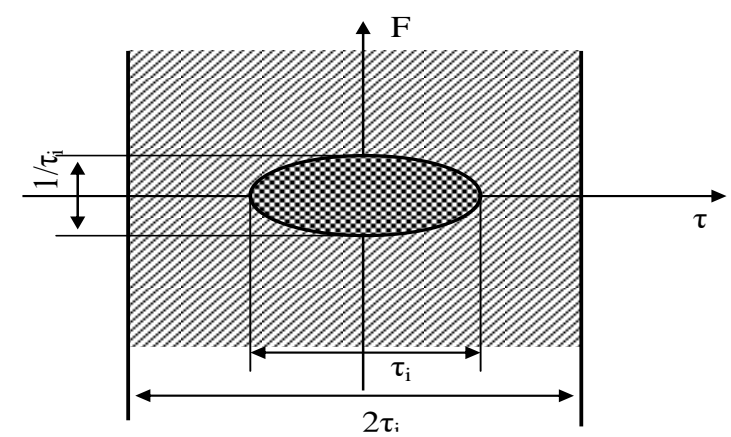

Figure 1. Horizontal slice of the surface of the radio pulse ambiguity function at 0.5 level

This is the curve of the form $|\sin (x) / x|$, which corresponds to its amplitude-frequency distribution. In Figure 3 the slice width $\tau=0$ is inversely proportional to the signal duration and determines the degree of frequency resolution (range rate) with coherent processing. The vertical profile $F=0$ see Figure 2(a), has the form of a triangle:

$$
\Psi(\tau, 0)= \begin{cases}1-|\tau| / \tau_{i}, & \tau \leq \tau_{i} \\ 0, & \tau>\tau_{i}\end{cases}
$$


The last expression shows that this is the matched-filter processing to the rectangular radio pulse. Its width at 0.5 level determines the time resolution. The plane graph of AF radio pulse without internal modulation is presented in Figure 3. Face perpendicular cuts $\tau=0$ and $F=0$ are shown in Figure 4. Face perpendicular cut $\tau=0$ narrows and $F=0$ expands when the pulse duration increases. The width of the correlation function of its complex envelope increases while compromising resolution when the pulse duration increases. This improves the ability to distinguish by Doppler-shift.

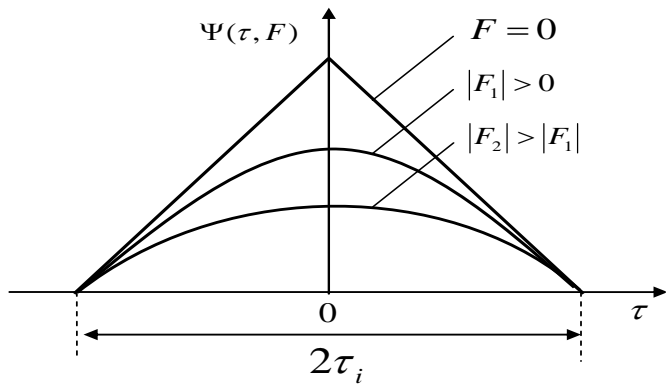

(a)

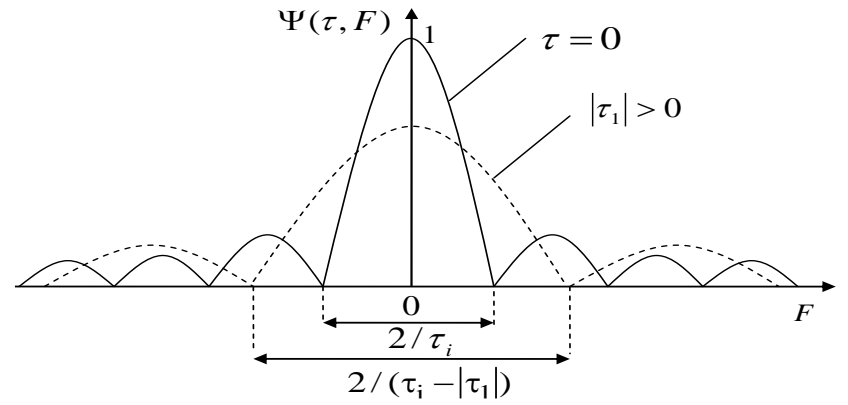

(b)

Figure 2. Vertical profile of the ambiguity function of the radio pulse from face perpendicular cut at: a) is $\tau=0, b)$ is $F=0$
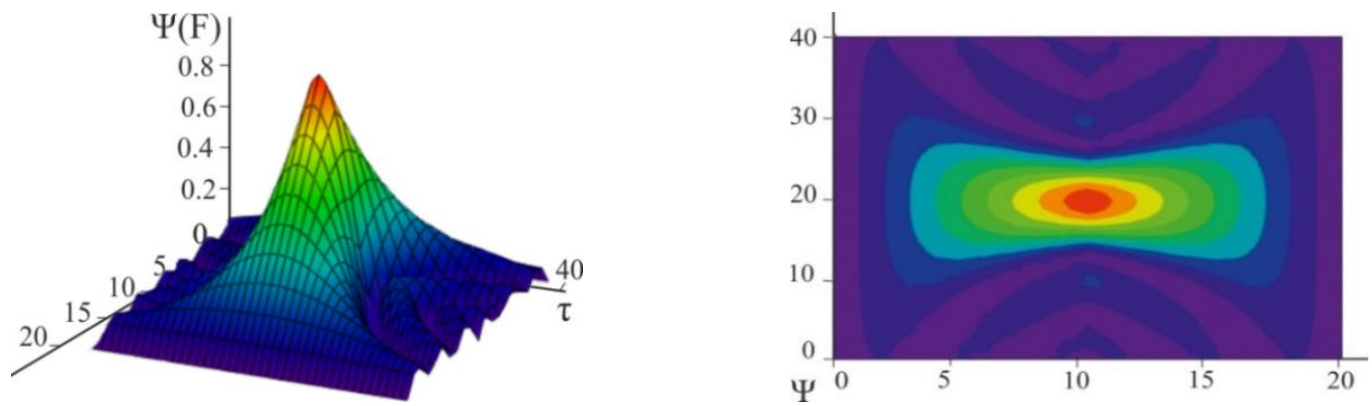

Figure 3. Vertical profile of the ambiguity function of the radio pulse from face perpendicular cut at $\tau=0$

Square and frequency-division signals can be taken as basis and used to determine the potential gain in terms of correlation efficiency. The value of the side lobes level can be determined by the algorithm:

a) to formulate the analytic $\mathrm{AF}$ expression $\Psi(\tau, F)$;

b) to find the derivate due to the corresponding coordinate $\mathrm{d} \Psi(\tau, F) / \mathrm{d} \tau$ or $\mathrm{d} \Psi(\tau, F) / \mathrm{d} F$;

c) to equate the derivate to zero and find the roots of equation;

d) to substitute the value of the root of equation in the AF expression.

According to the given algorithm, the target function of determining the optimal values of parameters can be formulated to achieve maximum gain.

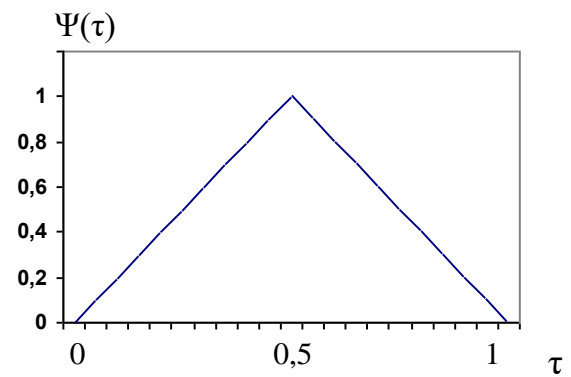

(a)

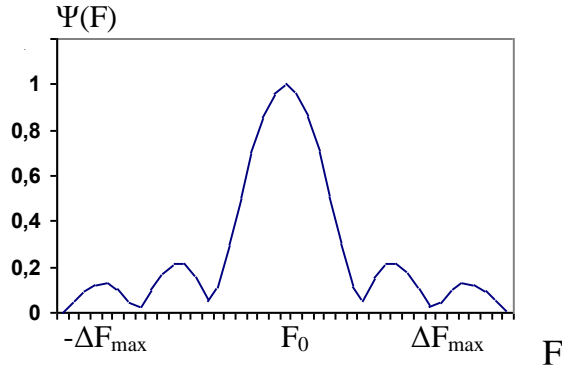

(b)

Figure 4. Face perpendicular cuts of ambiguity function simple radio pulse signal: a) is $\tau=0, b$ ) is $F=0$ 


\subsection{Analytical expressions of the ambiguity function taking into non-deterministic components}

Three cases of probing signals that are inherent in most of the pulsed high-frequency band generators are considered: the Gaussian amplitude envelope and frequency-division filling; rectangular amplitude envelope and linear frequency modulated filling; Gaussian envelope and linear frequency modulated filling; rectangular envelope and V-shaped non-linear frequency modulated filling.

In the first case, the signal model will have the form:

$$
U(t)=U_{0} k_{1} \exp \left(-\pi k_{1}^{2} t^{2}\right) \exp \left(j \omega_{0} t+\phi_{0}\right),|t| \leq 1
$$

where $k_{1} \exp \left(-\pi k_{1}^{2} t^{2}\right)$ is a component which instantiates the existing amplitude modulation in the form of a Gaussian envelope; $k_{1}=1 / \sqrt{ } 2 \pi \sigma$ is the amplitude distortion factor.

$\mathrm{AF}$ expression of such a signal model is obtained:

$$
\Psi(\tau, F)=\exp \left[-\frac{\tau^{2}}{4 \pi \sigma_{u}^{2}}-\pi^{3} \sigma_{u}^{2} F^{2}\right],|t| \leq \tau_{i}
$$

In the second case the signal model will have the form:

$$
U(t)=U_{0} \exp \left(j k_{2} t\right) \exp \left(j \omega_{0} t+\phi_{0}\right),|t| \leq \tau_{i}
$$

where $k_{2}=\beta t$ is a component that characterizes the available frequency modulation in the form of LFM [6]; $\beta$ is an angular distortions factor.

$\mathrm{AF}$ expression of such a signal model is obtained:

$$
\Psi(\tau, F)=\frac{\sin \left[(\beta \tau-\pi F)\left(\tau_{i}-|\tau|\right)\right]}{\tau_{i}(b \tau-\pi F)},|t| \leq \tau_{i}
$$

In the third case the signal model will have the form:

$$
U(t)=U_{0} k_{1} \exp \left(-\pi k_{2} t\right) \exp \left(j \omega_{0} t+\phi_{0}\right),|t| \leq \tau_{i}
$$

where $k_{1}$ and $k_{2}$ are components that characterize the existing amplitude and frequency modulations in the form of the Gaussian envelope and LFM respectively. After performing mathematical transformations the AF expression of a signal model is obtained in the form:

$$
\begin{gathered}
\Psi(\tau, F)=\exp \left[-\sigma_{u}^{2} \times\right. \\
\left.\times\left(\pi^{3} F^{2}+2 \pi^{2} \beta \tau F+4 \pi \beta^{2} \tau^{2}\right)-\frac{\tau^{2}}{4 \pi \sigma^{2}}\right], \quad|t| \leq \tau_{i}
\end{gathered}
$$

In the fourth case when there is LFM complex in the signal, the analytical expression describing the probing signal can be:

$$
U(t)=U_{0} \exp \left(j k_{2} t\right) \exp \left(j \omega_{0} t+\phi_{0}\right),|t| \leq \tau_{i}
$$

where:

$$
k_{2}= \begin{cases}\frac{a t}{2}, & t_{n} \leq t \leq t_{6} \\ -\frac{b t}{2}, & t_{6}<t \leq t_{3}\end{cases}
$$

The coefficients $a$ and $b$ are different because they describe the different dynamics of phase change, and moreover, the time intervals $t_{n} \leq t \leq t_{6}$ and $t_{6}<t<t_{3}$ may not be the same. In order to simplify the analysis process it is necessary to place the envelope vertex at the point $t=0$. So, the concepts of the time interval of 
frequency increase $\tau_{+}=t_{6}-t_{n}$ and the time interval of frequency fall $\tau_{-}=t_{3}-t_{6}$ are introduced. Accordingly, the expressions for determining the coefficients $a$ and $b$ will get the form:

$$
a=\frac{\pi \alpha \Delta I_{\phi}}{\tau_{+}}, \quad b=\frac{\pi \alpha \Delta I_{B}}{\tau_{-}}
$$

Take note the expressions (10) and (11):

$$
k_{2}=\left\{\begin{array}{lc}
a t, & -\tau_{+} \leq t \leq 0 \\
-b t, & 0<t \leq \tau_{-}
\end{array}\right.
$$

The expression for a signal with V-shaped reverse LFM modulation can be represented: $u(t)=u_{1}(t)+u_{2}(t)$ providing that $-\tau_{+} \leq t \leq \tau_{-}, u(t)$ for other $t$, where the signal components $u_{1}(t), u_{2}(t)$ are defined:

$$
\begin{aligned}
& u_{1}(t)=\frac{1}{\sqrt{2 \tau_{+}}} \exp \left(j a t^{2}\right),-\tau_{+} \leq t \leq 0 \\
& u_{2}(t)=\frac{1}{\sqrt{2 \tau_{-}}} \exp \left(-j b t^{2}\right),-\tau_{+} \leq t \leq 0
\end{aligned}
$$

AF consists of two autocorrelated terms $u_{1}(t), u_{2}(t)$ and two inter-autocorrelated terms corresponding to two mutual products $u_{1}(t)$ and $u_{2}(t)$.

$$
\psi(\tau, F)=\psi_{11}(\tau, F)+\psi_{12}(\tau, F)+\psi_{21}(\tau, F)+\psi_{22}(\tau, F)
$$

The main contribution is made by the autocorrelated terms $\Psi_{11}(\tau, F), \Psi_{22}(\tau, F)$ :

$$
\begin{gathered}
\Psi_{11}(\tau, F)=\frac{1}{2}\left(1-\frac{|\tau|}{\tau_{+}}\right) \frac{\sin \left[\pi\left(F-\frac{a \tau}{\pi}\right)\left(\tau_{+}-|\tau|\right)\right]}{\pi\left(F-\frac{a \tau}{\pi}\right)\left(\tau_{+}-|\tau|\right)} \times \\
\times \exp \left[j\left(\pi F\left(\tau_{+}+\tau\right)-a \tau \tau_{+}\right)\right], \quad-\tau_{+} \leq \tau \leq \tau_{-} \\
\Psi_{22}(\tau, F)=\frac{1}{2}\left(1-\frac{|\tau|}{\tau_{-}}\right) \frac{\sin \left[\pi\left(F+\frac{b \tau}{\pi}\right)\left(\tau_{-}-|\tau|\right)\right]}{\pi\left(F+\frac{b \tau}{\pi}\right)\left(\tau_{-}-|\tau|\right)} \times \\
\times \exp \left[j\left(\pi F\left(\tau_{-}+\tau\right)+b \tau \tau_{-}\right)\right], \quad-\tau_{+} \leq \tau \leq \tau_{-}
\end{gathered}
$$

Common expression for $\mathrm{AF}$ is:

$$
\begin{aligned}
\Psi(\tau, F) & =\left|\frac{1}{2}\left(1-\frac{|\tau|}{\tau_{+}}\right) \frac{\sin \left[\pi\left(F-\frac{a \tau}{\pi}\right)\left(\tau_{+}-|\tau|\right)\right]}{\pi\left(F-\frac{a \tau}{\pi}\right)\left(\tau_{+}-|\tau|\right)}\right|+ \\
& +\left|\frac{1}{2}\left(1-\frac{|\tau|}{\tau_{-}}\right) \frac{\sin \left[\pi\left(F+\frac{b \tau}{\pi}\right)\left(\tau_{-}-|\tau|\right)\right]}{\pi\left(F+\frac{b \tau}{\pi}\right)\left(\tau_{-}-|\tau|\right)}\right|
\end{aligned}
$$




\section{RESULTS AND ANALYSIS}

The analysis of the received AF allows to establish that there is a quadratic dependence of the correlation function module of the complex envelope depending on the amplitude envelope dispersion or on the angle modulation index in each of them. This means that there are certain extreme values of these quantities which determine their correlation properties in the signals synthesis with diven complex envelope and in the algorithms consistent for their processing.

\subsection{Analysis of received expression of ambiguity functions}

An extreme value is obtained in relation of the dispersion to the pulse duration where the gain value by the distinction on the frequency and on the time axis are the same [8]. Deviation from this value in one direction leads to a sharp increase in the time-delay gain and in the other direction-in Doppler-shift as it is illustrated in Figure 5(a-c).

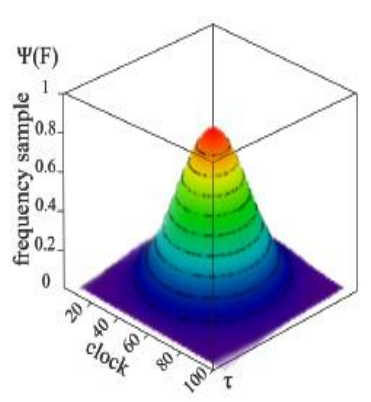

(a)
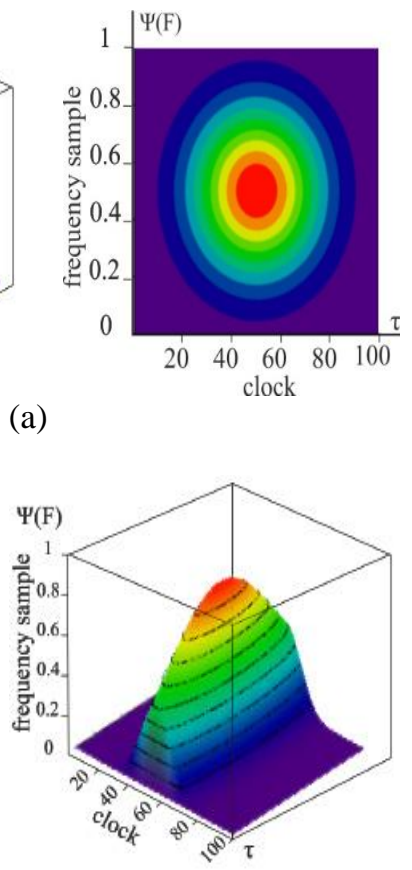

(c)

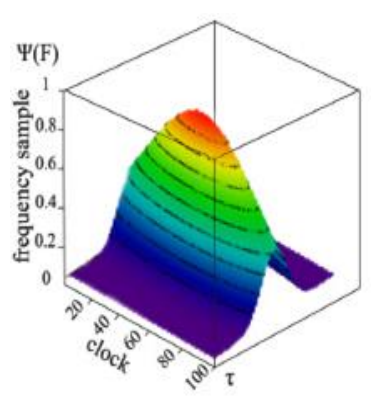

(b)
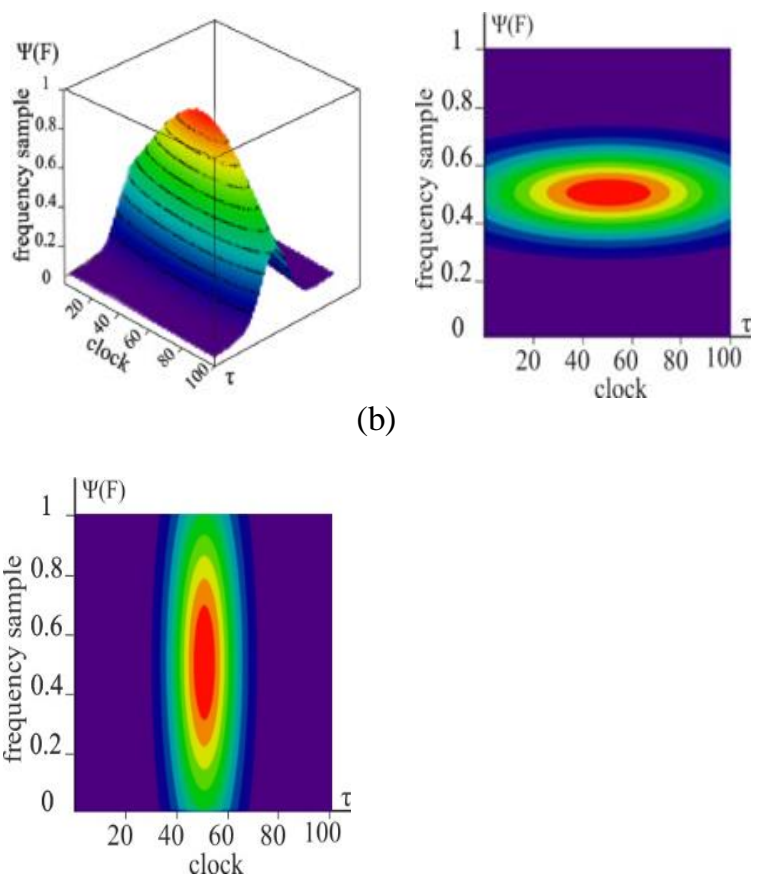

.

Figure 5. Ambiguity function view of pulse radio signal taking gaussian amplitude envelope form: a) is $\left.\tau_{\mathrm{i}} / \sigma_{\mathrm{u}}=2 \pi, \mathrm{b}\right)$ is $\left.\tau_{\mathrm{i}} / \sigma_{\mathrm{u}}=\pi, \mathrm{c}\right)$ is $\tau_{\mathrm{i}} / \sigma_{\mathrm{u}}=3 \pi$

The presence of amplitude and frequency transformations in the signal are considered. The simulation results of the obtained expression for an AF radar Gaussian signal and a LFM (chirp) are shown in Figure 6 (a)-(e). A significant decrease of relative gains due to pointed ratios of instabilities and in the presence of the Doppler-shift are presented in Figure 7. Figure 8 demonstrates graphs of relationship between specific width of correlation function main lobe for complex signal envelope and amplitude envelope dispersion. Various values of the angle modulation index were selected $[9,10]$.

In the implementation of the autocorrelation estimation algorithm, the defined approach is proposed in constructing of LFM structures with digital signal processing. ADC is applied in the intermediate frequency sections - to the detector. In this case the received signal is discretized immediately after spatial discrimination in a sufficiently wide band of frequencies [11-13]. The amplification and reduction of the carrier frequency is performed to a value that is convenient for the further processing. In this option, all operations can be implemented with digital processing including frequency-time processing of the signal by one probing [10]. Advantages of this approach are: optimal single- and multichannel frequency-time filtration of signals; measuring coordinates that correspond to the detected signals; calculation of error signals by angular coordinates, range and speed for the tracking capability. The Figure 9 shows the signal surface of the AF obtained in accordance with the equation (18) with V-shaped asymmetric linear frequency modulation. 


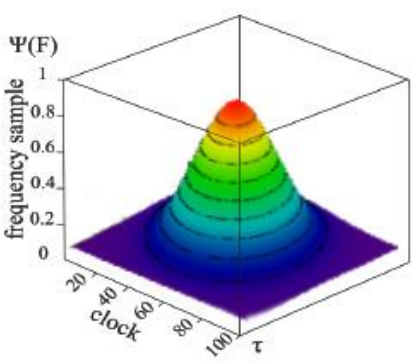

(a)
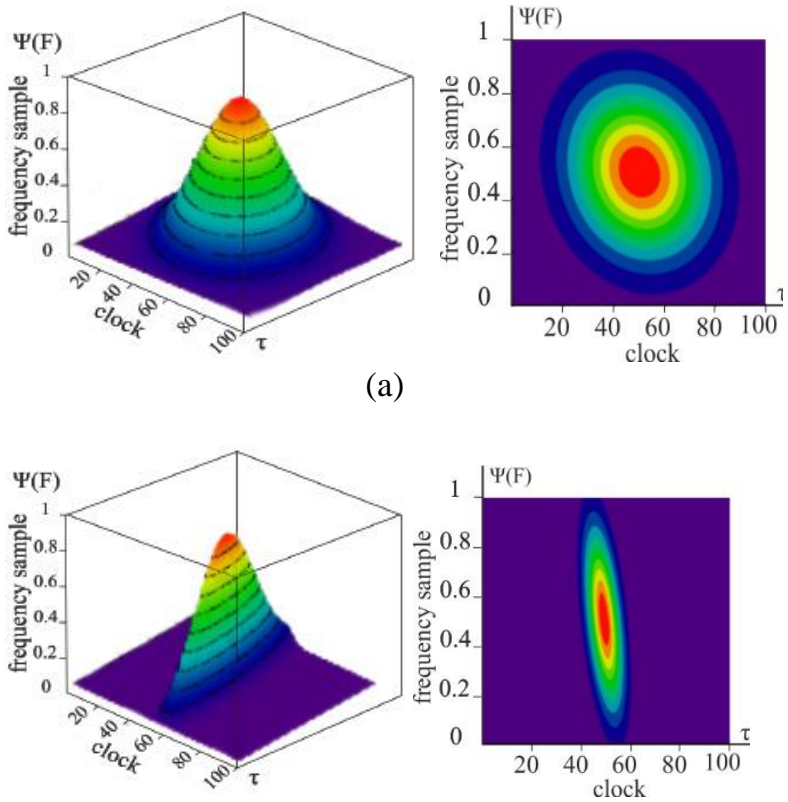

(c)

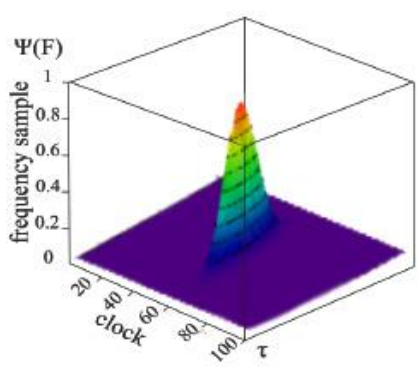

(e)
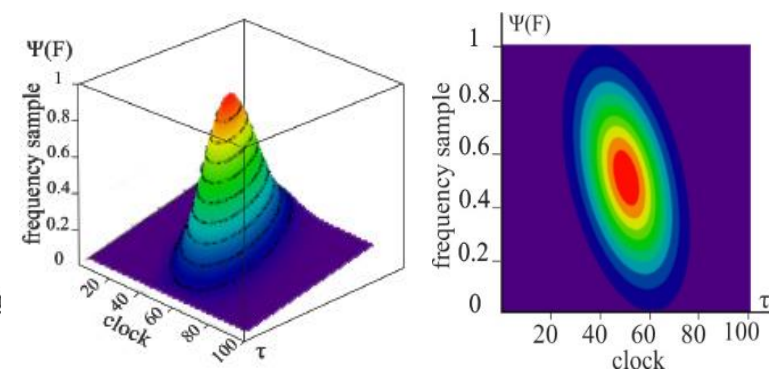

(b)
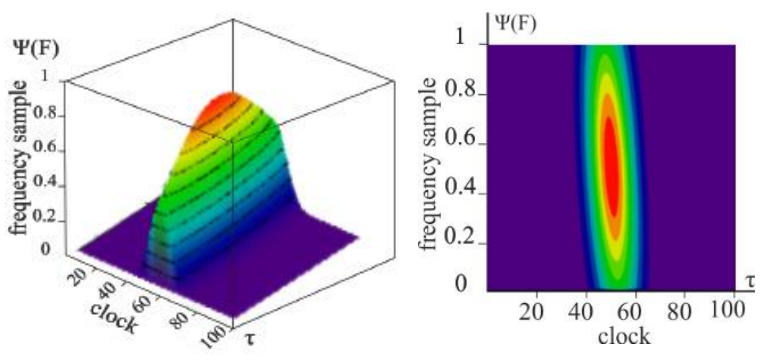

(d)

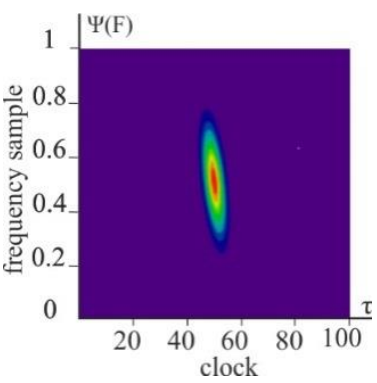

Figure 6. Ambiguity function view of pulse radio signal taking Gaussian amplitude envelope form and LFM: a) is $\left.\beta \tau_{i}^{2}=1, \tau_{i} / \sigma_{u}=2 \pi, \mathrm{b}\right)$ is $\left.\beta \tau_{i}^{2}=5, \tau_{i} / \sigma_{u}=2 \pi, \mathrm{c}\right)$ is $\beta \tau_{i}^{2}=15, \tau_{i} / \sigma_{u}=2 \pi$,

d) is $\beta \tau_{i}^{2}=15, \tau_{i} / \sigma_{u}=4 \pi$, e) is $\beta \tau_{i}^{2}=15, \tau_{i} / \sigma_{u}=\pi$

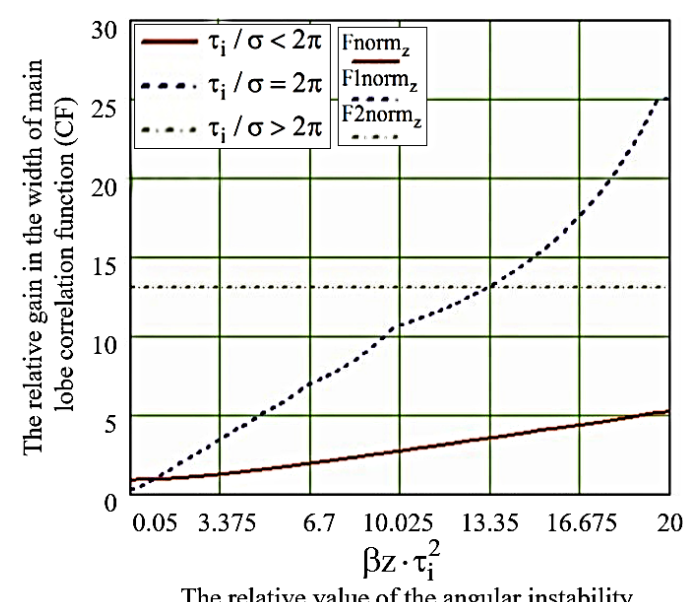

The relative value of the angular instability

(a)

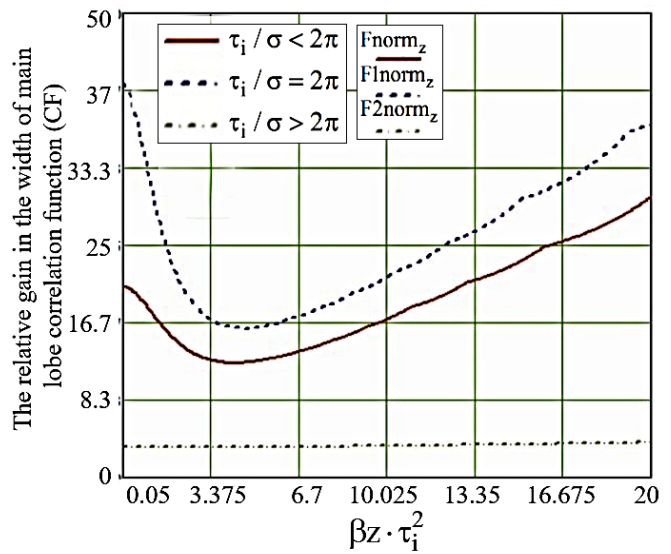

The relative value of the angular instability

(b)

Figure 7. Dependence of the relative gain in the main lobe width of the correlation function of pulse radio signal taking into account the Gaussian form of the amplitude envelope and LFM from angular instability in different frequency sections $(a, b)$ 


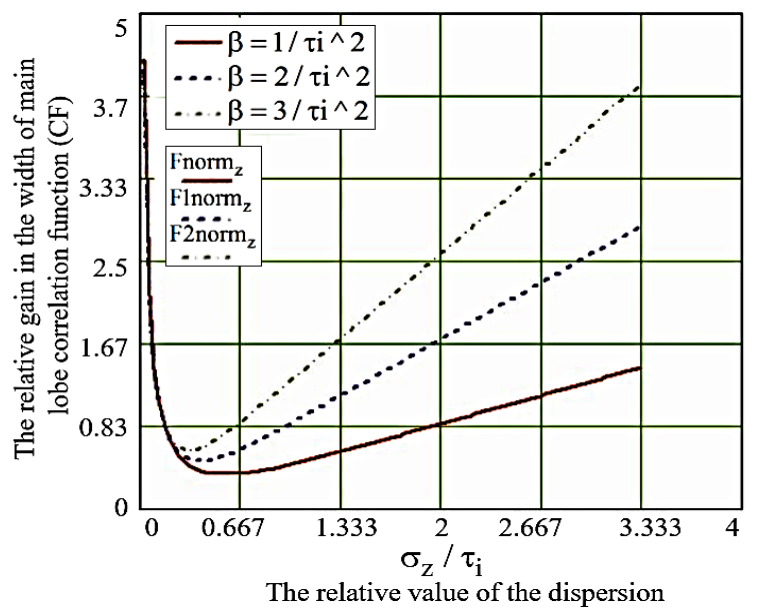

(a)

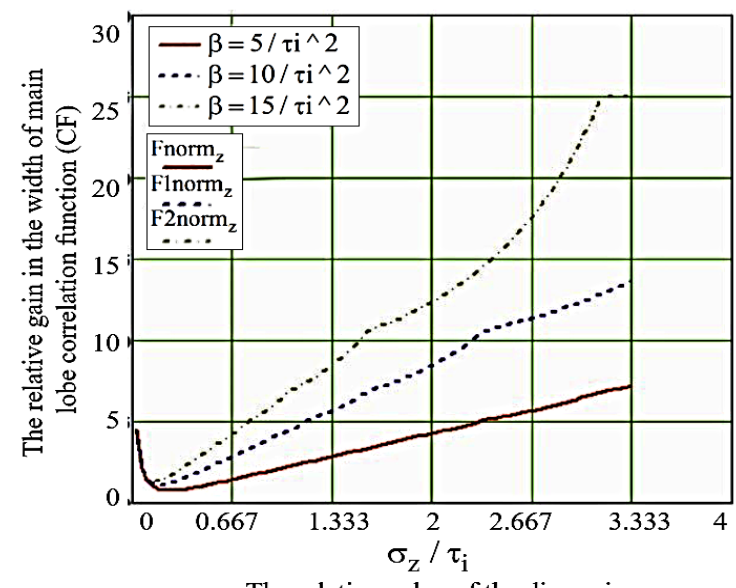

The relative value of the dispersion

(b)

Figure 8. Dependence of the relative gain in the main lobe width of the pulsed radio signal correlation function and LFM depending on the amplitude envelope dispersion at different values of the anglemodulation index $(a, b)$

The width of the main lobe is equal to the double minimum period of frequency instability at a cut of 0.5 Figure 9 (c). The higher frequency of instability (for LFM is frequency deviation) makes the main lobe cut become shorter. There are two "ridges" placed at $\alpha$ and $\beta$ angles to the vertical plane cut $F=0$ unlike the AF of the usual LFM signal. Obviously, the magnitude of these angles depends on the value $a / \tau_{+}$and $b / \tau_{\text {.. }}$ They will be larger the more these ratios are larger so, the more frequency deviation is greater. The expression (18) is valid just for pointed case because of the restrictions imposed on the incoherence of the envelope regions signal to $t_{b}$ and after it. If the signal is more complicated or these areas are interconnected, the AF will take the following form (19).

$$
\begin{gathered}
\Psi_{12}(\tau, F)=\frac{1}{\left(\tau_{+}+\tau_{-}\right) \sqrt{a}} \operatorname{rect}\left(\frac{\tau}{\left(\tau_{+}+\tau_{-}\right)}-\frac{1}{2}\right) \times \\
\times \exp \left(\frac{-j \pi a\left(\tau_{+}-\tau\right)^{2}}{2}\right) \exp \left(\frac{j \pi F^{2}}{(a+b)}\right)\left[Z^{*}\left(x_{1}\right)+Z^{*}\left(x_{2}\right)\right] \\
x_{1}=\tau \sqrt{a}+\frac{F}{\sqrt{a}}, \quad x_{2}=\tau \sqrt{b}+\frac{F}{\sqrt{b}}
\end{gathered}
$$

where $\mathrm{Z}(\mathrm{x})$ is a Fresnel complex integral.

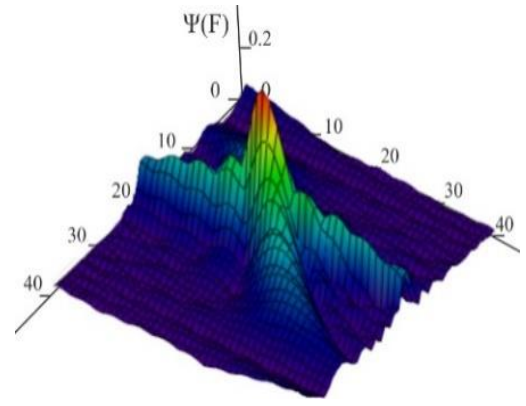

(a)

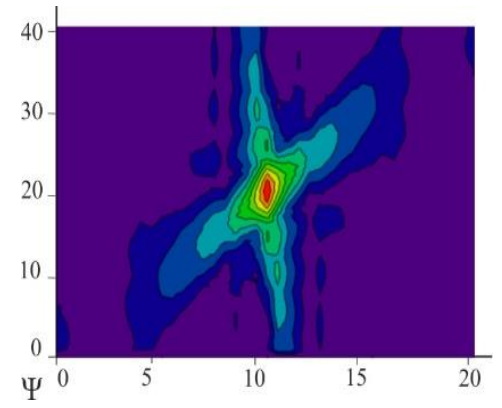

(b)

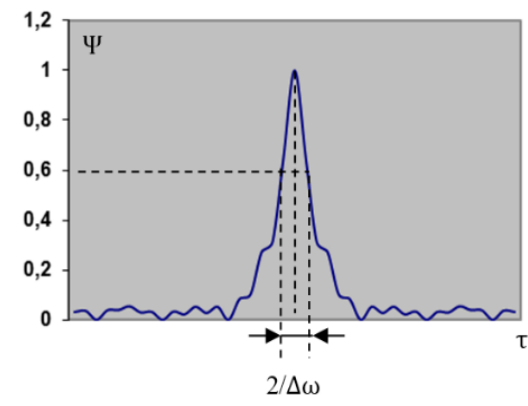

(c)

Figure 9. The ambiguity function surface of pulsed radio signal taking into account internal phase-frequency instability: a) is the function surface, b) is the function projection, c) is the ambiguity function width in the vertical plane cut $F=0$ 
Table 1 gives a comparative characteristic of the width of ambiguity function at 0.5 for different values of the maximum instability frequency. As can be seen from the table, the higher the deviation frequency, the greater the compression ratio at the matched filter output. This is easy to explain since the width of the main sheet for a simple chirp pulse is calculated through its base, $B=\Delta f T_{\mathrm{c}}$.

Table 1. Comparative characteristic of the ambiguity function parameters

\begin{tabular}{cccccc}
\hline$\Delta f_{+}$ & $\Delta f$. & $\tau_{+}$ & $\tau$ & The main leaf width at the level of 0,5 & Side leaf level \\
\hline \multirow{6}{*}{$10^{7}$} & & 0 & 0 & 0.242 & -20 \\
& & $0.1 \tau_{\mathrm{i}}$ & $0.9 \tau_{\mathrm{i}}$ & 0.15 & -26 \\
& $0.2 \tau_{\mathrm{i}}$ & $0.8 \tau_{\mathrm{i}}$ & 0.175 & -23 \\
& & $0.3 \tau_{\mathrm{i}}$ & $0.7 \tau_{\mathrm{i}}$ & 0.175 & -25 \\
& $0.4 \tau_{\mathrm{i}}$ & $0.6 \tau_{\mathrm{i}}$ & 0.167 & -25.84 \\
& $0.5 \tau_{\mathrm{i}}$ & $0.5 \tau_{\mathrm{i}}$ & 0.155 & -24.9 \\
$8 \cdot 10^{6}$ & 0 & $\tau_{\mathrm{i}}$ & 0.43 & -14.8 \\
& & $0.1 \tau_{\mathrm{i}}$ & $0.9 \tau_{\mathrm{i}}$ & 0.171 & -20 \\
& & $0.2 \tau_{\mathrm{i}}$ & $0.8 \tau_{\mathrm{i}}$ & 0.256 & -15 \\
& $0.3 \tau_{\mathrm{i}}$ & $0.7 \tau_{\mathrm{i}}$ & 0.252 & -18 \\
& $0.4 \tau_{\mathrm{i}}$ & $0.6 \tau_{\mathrm{i}}$ & 0.229 & -20 \\
\hline
\end{tabular}

\subsection{Results of structural and schematic solutions for the application of the developed method}

When implementing the algorithm of estimating radar signals in the entire receiver bandwidth and taking into account the angular and amplitude fluctuations, the decisive criterion of efficiency is the quality of their transformation into digital form. At this processing stage all signal components that are outside of the known values are lost (maximum allowable deviation of frequency, amplitude, phase). In most modern radar systems analog-to-digital conversion is performed after the detector [10-22].

When implementing the autocorrelation estimation algorithm, it is necessary to apply a different approach in the construction of radar structures with digital signal processing, namely the application of ADC in the intermediate frequency path-to the detector. In this case, the received signal is sampled immediately after spatial selection in a sufficiently wide frequency band, amplifying and reducing the carrier frequency to a value that is convenient for further processing. In this embodiment, it is possible to perform all operations in digital processing including frequency-time signal processing [23].

To implement the method which is proposed in this work, it is necessary to organize the operation of the signal processing device for digital processing in such a way as to ensure the fulfillment of both the listed tasks and additional ones, which are in the selection of echo-signals by the correlation features properties of their complex bypass, and therefore only quadratic ADC implementation or detection [24-26]. Signal processing device on the basis of a programmable processor consists of two interrelated parts as shown in Figure 10, the device of gain and signal conversion and the actual programmable signal processor. Figure 11 presents flowchart of the signal amplification and conversion channel.

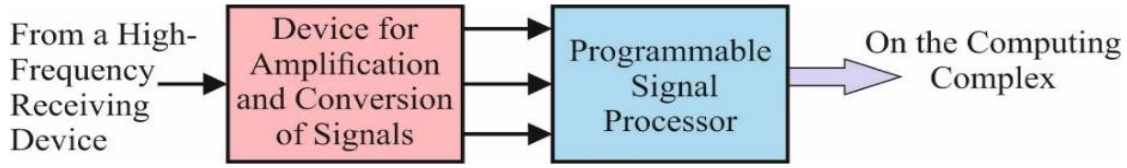

Figure 10. Structural diagram of radar with digital signal processing to detection

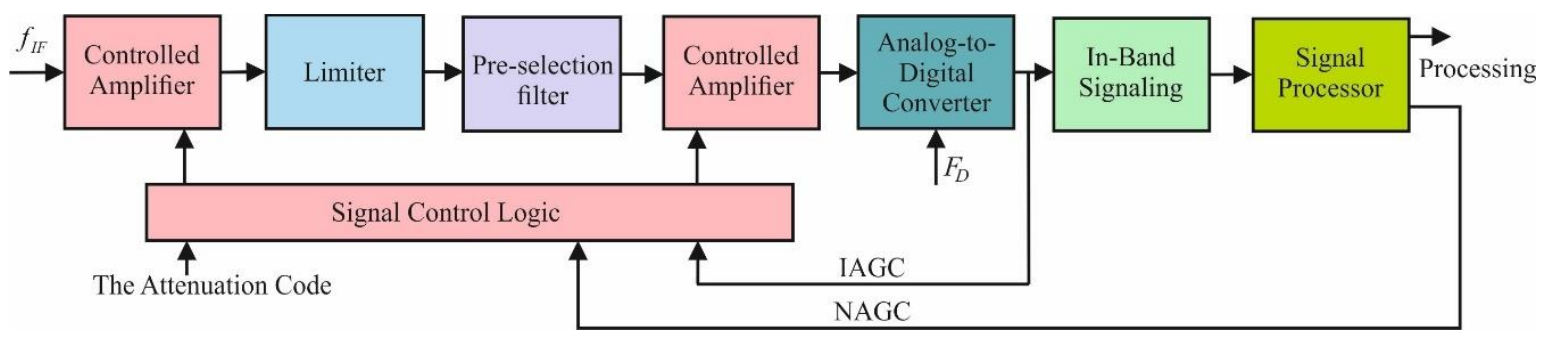

Figure 11. Flowchart of the signal amplification and conversion channel: $F_{D}$ - sampling rate; $f_{I F}$ - intermediate frequency 
The principles of the first adjustment as shown in Figure 11, (Noise Automatic Gain ControlNAGC) are as follows. The timing diagram of the radar periodically (for example, once in a few minutes) highlights the special clock for the considered adjustment. No radar is emitted at this clock and the signal processing path operates at its own noise. In this case, the signal processor evaluates the value of the ratio $\sigma_{I N} / h$ ( $\sigma_{I N}$-rms input noise; $h$ - quantization step) of the ADC source codes and calculates the necessary change in the gain.

The second adjustment is due to the fact that for the normal operation of the device the signal must be within the linear part of the amplitude characteristic (within the dynamic range of noise), for quasi-continuous signals gain tuning may be performed at the beginning of the sounding cycle. In this case, the gain reduction command shall be made by a special scheme at the output of the ADC according to the results of source code analyzes for the pulse repetition period. This procedure is repeated cyclically during the first few repetition periods (instantaneous automatic gain control - IAGC). Finally, the position can significantly improve the limiter before the pre-selection filter. The restriction level should correspond to the restriction level in the ADC. The transfer of the restriction to the point of the path to the filter allows the selection of the resulting harmonics significantly reduce their negative impact with the help of filter.

\section{CONCLUSION}

Internal instability interpretation as V-shaped LFM in the synthesis of AF analytical expression is the most appropriate. Studies have shown that as higher the deviation frequency as the signal compression degree at the matched filter output is greater. This is due to the fact that the main lobe width for LFM simple pulse is calculated through its base. Numerical simulation of the obtained AF expressions showed that taking into account the fluctuation components in a complex envelope incoherent radar signal enables to increase its potential for differentiation (both by frequency and by time-delay). The gain can reach several times without distribution amplification signal by providing the optimal correlation between the parameters of amplitude and angular fluctuations.

The analytical expressions of AF radio signals modulated by narrowband random processes with different distribution laws are the first to propose in the paper. The novelty of the obtained expressions is to take into account the distribution laws of simultaneously acting amplitude and angular fluctuations with a limited interval of change of their parameters in the complex model's envelope of radar signals. The proposed in paper approach gives a definite system advantages: stability of characteristics throughout the range of operating conditions; the adapt ability to changing working conditions; device upgrading by modifying the software without changing the hardware; reduction of weight, dimensions and as a consequence, a significant reliability increase; easy configuration of equipment; reduced price compared with the analog variant due to the greater processability and low prices of the components in the wide production.

\section{REFERENCES}

[1] I. R. Parhomey, J. M. Boiko, and O. I. Eromenko, "Features of digital signal processing in the information control systems of multipositional radar," Journal of Achievements in Materials and Manufacturing Engineering, vol. 77, no. 2, pp. 75-84, 2016.

[2] J. Lee, and H. Bang, "Radial basis function network-based available measurement classification of interferometric radar altimeter for terrain-aided navigation," IET Radar, Sonar \& Navigation, vol. 12, no. 9, pp. 920-930, 2018.

[3] T. J. Daim, and R. M. A. Lee., "A weighted least squares consideration for IR-UWB radar based device-free object positioning estimation for indoor environment," Indonesian Journal of Electrical Engineering and Computer Science (IJEECS), vol. 15, no 2, pp. 894-901, 2019.

[4] S. Savastano, and R. Guida, "Uncertainty quantification in synthetic aperture radar remote sensing data processing," Proc. IEEE International Geoscience and Remote Sensing Symposium, pp. 9193-9196, 2018.

[5] J. Wang, B. Zhang, and P. Lei, "Ambiguity function analysis for OFDM radar signals," Proc. IEEE CIE International Conference on Radar, pp. 1-5, 2016.

[6] M. G. M. Hussain, "Ambiguity function of bipolar ultrawideband-throb signal," Proc. IEEE Radar Conftrence, pp. 0376-0381, 2014.

[7] C. Hu, et al., "Generalized ambiguity function properties of ground-based wideband MIMO imaging radar," IEEE Transactions on Aerospace and Electronic Systems, vol. 55, vol. 2, pp. 578-591, 2019.

[8] J. Nieh, and R. A. Romero, "Comparison of ambiguity function of eigenwaveform to wideband and pulsed radar waveforms: a comprehensive tutorial," The Journal of Engineering, vol. 2018, no. 4, pp. 203-221, 2018.

[9] S. J Rosli, H. A. Rahim, and K. N. Abdul Rani, "Design of amplitude and phase modulated pulse trains with good auttocorrelation properties for radar communications," Indonesian Journal of Electrical Engineering and Computer Science (IJEECS), vol. 13, no 3, pp. 990-998, 2019.

[10] H. Xu-Dong, et al., "Research on multi-target resolution process with the same beam of monopulse radar," Proc. IEEE 17th International Conference on Communication Technology, pp. 1102-1105, 2017. 
[11] J. Boiko, and O. Eromenko, "Signal processing in telecommunications with forward correction of errors," Indonesian Journal of Electrical Engineering and Computer Science (IJEECS), vol. 11 no. 3, pp. 868-877, 2018.

[12] J. Boiko, et al, "Signal processing with frequency and phase shift keying modulation in telecommunications," TELKOMNIKA Telecommunication, Computing, Electronics and Control, vol. 17, no. 4, pp. 2025-2038, 2019.

[13] S. Suparman, and M. Doisy, "Bayesian segmentation in signal with multiplicative noise using reversible jump MCMC," TELKOMNIKA (Telecommunication, Computing, Electronics and Control), vol. 16, no. 2, pp. 673-680, 2018.

[14] U. Kaydok, "Baseband signal modelling of chaff echoes for coherent pulsed radars," Proc. IEEE Radar Conference, pp. 0642-0646, 2018.

[15] D. Schvartzman, and C. D. Curtis, "Signal processing and radar characteristics (SPARC) simulator: A Flexible dual-polarization weather-radar signal simulation framework based on preexisting radar-variable data," IEEE Journal of Selected Topics in Applied Earth Observations and Remote Sensing, vol. 12, no. 1, pp. 135-150, 2019.

[16] T. Rogala, A. Kawalec, and M. Szugajew, "Application of CUDA computing technology in radar digital signal processing," Proc. 2016 17th International Radar Symposium, pp. 1-4, 2016.

[17] J. Boiko, I. Kovtun, and S. Petrashchuk, "Productivity of telecommunication systems with modified signal-code constructions," Proc. 4th IEEE International Scientific-Practical Conf. Problems of Infocommunications. Science and Technology, pp. 173-178, 2017.

[18] R. Sedletsky, "The stationary phase method calculating amplitude modulation for a pseudo random signal with a small side lobe level of correlation function," Proc. 2015 16th International Radar Symposium, pp. 1165-1170, 2015.

[19] P. K. Mane, and K. N. Rao, "Granular mobility-factor analysis framework for enriching occupancy sensing with doppler radar," International Journal of Electrical and Computer Engineering (IJECE), vol. 8, no. 2, pp. 979-988, 2018.

[20] M. Lahsaini, L. Zenkouar, and S. Bri, "Modeling of a Microwave Amplifier Operating around $11 \mathrm{GHz}$ for Radar Applications," International Journal of Electrical and Computer Engineering (IJECE), vol. 8, no. 5, pp. 979-988, 2018.

[21] L. Zhang, "A high resolution imaging method for sweep frequency continuous wave radar," Proc. 2016 CIE International Conference on Radar, pp. 1-4, 2016.

[22] Y. Deng, et al., "Cascaded suppression method for airborne passive radar with contaminated reference signal," IEEE Access, vol. 7, pp. 50317-50329, 2019.

[23] J. Yan, et al., "Joint threshold adjustment and power allocation for cognitive target tracking in asynchronous radar network," IEEE Transactions on Signal Processing, vol. 65, no. 12, pp. 3094-3106, 2017.

[24] H. Liu, et al., "Tracking-aided signal fusion detection method," Proc. 2016 CIE International Conference on Radar, pp. 1-4, 2016.

[25] R. Zeng, et al., "Joint estimation of frequency offset and doppler shift in high mobility environments based on orthogonal angle domain subspace projection," IEEE Transactions on Vehicular Technology, vol. 67, no. 3, pp. 2254-2266, 2017.

[26] Y. Liu, et al., "Projection techniques for altitude estimation over complex multipath condition-based VHF radar," IEEE Journal of Selected Topics in Applied Earth Observations and Remote Sensing, vol. 11, no. 7, pp. 2362-2375, 2018. 